\title{
THE HIGHEST ENERGY COSMIC RAYS
}

\author{
COLIN A. NORMAN \\ Dept. of Physics and Astronomy - Johns Hopkins University \\ and \\ Space Telescope Science Institute - 3700 San Martin Drive \\ Baltimore, MD 21218
}

\begin{abstract}
The current data on the highest energy cosmic rays (UHECRs) is discussed and an understanding of the origin of these particles is reviewed. New and proposed facilities for measurement of UHECRs, neutrinos and $\gamma$ rays can interestingly and significantly constrain the physics of the source origin. Cosmic magnetic field strengths are the most uncertain physical parameter.
\end{abstract}

\section{Introduction}

Great new data on UHECRs from the Fly's Eye air shower array experiment indicates that at about $10^{18.5} \mathrm{eV}$ there is both a change in the slope of the energy spectrum and also a change in the composition of the incoming high energy particles from heavy ( $\mathrm{Fe}$ ) to light $(\mathrm{p})$ with increasing energy (Bird et al., 1995, Gaisser 1993). This naively looks like the signature of the extragalactic component accelerated from essentially primordial material and after detailed analysis (Norman, Melrose and Achterberg 1995, hereafter NMA) we argue that this is a solid conclusion. However, recent data from the Akeno group on the muonic component ( $\gtrsim \mathrm{GeV}$ ) for UHECR generated events does not find the expected decrease in the muon to electron ratio as the composition of UHECRs changes from Iron to protons, possibly indicating a smaller composition change than that inferred from the Fly's Eye experiment.

Previous theories of UHECRs have fallen into two classes: (1) Extragalactic Shocks pioneered by Cavallo (1978) and developed in detail by Axford (cf. Axford 1991) and Biermann and Stanev and collaborators (Rachen 
et al., 1993, Stanev et al., 1995); and (2) Active Galactic Nuclei most recently elegantly discussed by Protheroe and Szabo (1992) and Szabo and Protheroe (1994) and Stecker, Done, Salamon and Sommers (1991). We show here that the radiation field in AGN limits the maximum energy and the propagation length for photopion production to values that exclude AGNs as source for UHECRs. New ideas about the origin of UHECRs include large scale shocks associated with cosmic structure formation (NMA, Kang, Ryu and Jones 1995) and cosmological gamma-ray bursts (Vietri, 1995, Waxman 1995, Milgrom and Usov 1995a,b).

General constraints on the origin of UHECRs were presented in the influential papers of Hillas (1984) and Hill and Schramm (1985) and more recently by Sigl, Schramm and Bhattacharjee (1995) and NMA. Detailed analysis of the current data of the energy spectrum concerning the existing of the GZK cut-off or not and also the implications for a potential new source of UHECRs due to physical phenomena associated with, for example, topological defects associated with cosmic phase transitions have been analyzed by Yoshida and Teshima (1993), Bhattacharjee, Hill and Schramm (1992), Sigl, Lee, Schramm and Bhattacharjee (1995) and Waxman (1995).

One remark about symbolic notation in this paper. Where symbols have thir common or obvious meanings they are not defined due to space constraints. If the reader faces continuing difficulties then they should refer to Norman, Melrose and Achterberg (1995) or Norman and Lacey (1996).

\section{Metallicity-Composition Constraints}

The protonic component at the highest energies can have two explanations: (1) The source is unenriched or (2) the UHECRs can be accelerated from, say, a plasma of normal, solar metallicity composition and then the heavies can be selectively removed by photo-dissociation into protons on the cosmic background radiation field. Possibility (2) seems to be excluded for the smooth component extending up to $10^{19.5} \mathrm{eV}$ since at approximately $10^{19.5} \mathrm{eV}$ the proton energy loss rate is equal to the photodissociation rate for heavies and there is no obvious break in the energy or composition spectrum. However, this metallicity composition argument can be subject to increasingly detailed check as more data on UHECRs becomes available. We conclude that possibility (1) is the most reasonable and consequently rule out an origin of UHECRs in rich clusters, the metal rich environments of galaxies and other interesting models such as the metal-rich environment of colliding galaxies resulting in starbursts (Cesarsky and Ptuskin 1992).

The sources must be local $(z \lesssim 0.3)$ since there is a smooth spectrum up to $10^{19.5} \mathrm{eV}$ and protons cannot travel more than $\lesssim 1 \mathrm{Gpc}$ due to pair production and pion production on the cosmic radiation background. Extended 
halos around galaxies have often been invoked but the type of extended halo envisioned by Jokipii and Morfill (1985) has not been observed in general (cf. Wielebinski et al., 1995) although there are interesting special cases such as NGC 4631(Donahue, Aldering and Stocke 1995).

\section{UHECRs Cannot Originate in AGN}

At first, AGNs seem promising acceleration sites for UHECRs since if one assumes that there is rough equipartition between radiation energy density and magnetic field energy density in AGN (Rees 1987) the maximum energy is $E_{\max } \sim 3 \times 10^{19} \quad \beta_{-1} \quad Z \quad L_{46} \mathrm{eV}$ with standard meaning for the symbols (cf. NMA). Generally, AGNs have an intense inner radiation field well parameterized by the compactness parameter $l \sim\left(\sigma_{T} L\right) /\left(4 \pi m_{e} c^{3} R\right)$ which is generally $l \gtrsim 1$ (Done and Fabian 1989). Then the optical depth for photopion production in IR photons is $\sim 10^{3} l$ and the corresponding energy threshold is $\sim 10^{16} \mathrm{eV}$. Therefore, UHECRs above $10^{16} \mathrm{eV}$ do not escape AGN. In addition, the isotopic component within the BLR/warped disk/obscuring torus region is of order $10 \%$ of the predominately radial photon flow which inhibits use of any models with small angle between photon and particle motion (NMA).

A mention of neutrino astronomy in passing. Although models of neutrino production in AGN are rather uncertain there is a significant prospect that neutrino telescopes will at least place significant constraints on conditions in AGN. The Frejus experiment can already rule out certain classes of models as discussed by Mannhein (1995) for radio quiet AGN. It is not really important what the specifics of these rather uncertain models are, but what is important is that AMANDA, DUMAND, NESTOR and other neutrino facilities in various stages of implementation can easily increase the Frejus limit by orders of magnitude giving potentially quite significant constraints on neutrino producing regions in AGNs (cf. Gaisser, Halzen and Stanev 1995).

\section{UHECRs Can Originate in Extragalactic Shocks}

The maximum energy achievable in a standard Sedov-Taylor blast wave is $\sim 5 \times 10^{19} Z \quad B_{-6} \quad E_{61}^{2 / 5} \rho_{-31}^{-2 / 5} t_{8}^{-1 / 5} \ln R$ and in a wind blowing out into the IGM $\sim 10^{20} Z \quad B_{-6} \quad L_{46}^{2 / 5} \rho_{-31}^{-2 / 5} \quad t_{8}^{1 / 5} \mathrm{eV}$. The principal uncertainty here is the magnetic field strength and we have assumed here that microgauss field strengths can be self-generated in such shocks. Primordial field strengths are probably too low to be useful as discussed later.

FRII class radio sources have been frequently discussed as sources of UHECRs (Rachen et al., 1993) and there are places in a typical FRII 
source where UHECRs can be produced-the hot spot, and the extended cocoon shock. Analysis in NMA gives maximum energies of $\sim 10^{20} B_{-4} \beta_{j}$ $R_{j, 1 \mathrm{kpc}} \mathrm{eV}$, and $\sim 5 \times 10^{19} \mathrm{Z} B_{-6} L_{46}^{1 / 2} t_{8}^{1 / 2} n_{-4}^{-1 / 2} R_{10 \mathrm{kpc}} \mathrm{eV}$ respectively. These work well, however, FRII sources are generally found at $z \gtrsim 0.3$ and are quite rare within the allowed range of distances for UHECR. More detailed statistical analysis may eventually rule out FRIIs as a plausible source.

\section{UHECRs Can Originate from Cosmic Structure Formation}

We have analyzed the shocks resulting from cosmic structure formation at the current epoch using a typical CDM model with a typical scale $R_{S} \sim\left(2 G M / \Omega \delta H^{2}\right)^{\frac{1}{3}}$ and shock velocity $V_{s} \sim\left(2 G M / R_{s}\right)^{1 / 2}$ where $\delta \sim 178$ is the cosmical over density parameter after collapse and virialization of a perturbation that has grown to become non-linear. Having a typical mass scale of $M \sim 10^{15}(0.4 / b(1+z))^{\frac{6}{n+3}} M_{\odot}$ where $n$ is the index of the intial fluctuation spectrum $\left(\left|\delta_{k}\right|^{2} \propto k^{n}\right)$ we find a maximum energy of $\sim 3 \times 10^{18} Z B_{-6} \Omega^{-1 / 6} h^{-1 / 3}(1+z)^{-5 / 2} \mathrm{eV}$ where again we have assumed self-generated magnetic field strengths of order micro-gauss-consistent with values observed in clusters. Independent of CDM models, typical, collapsing, pancake-like objects give similar estimates of $E_{\text {max }}$. The fluxes expected from these structures are consistent with the observed fluxes of UHECRs. Similar work has been done in this context by Kang, Ryu and Jones (1996).

It is most interesting then that Stanev et al., (1995) have found tentative evidence for a correlation of the arrival direction of the highest energy cosmic rays with very roughly the direction of the super galactic plane. Many selection effects come into play and the exact statistical significance is rather uncertain but it is a most interesting suggestion that follows earlier work of Wdowczyk and Wolfendale (1979) and Giler, Wdowczyk and Wolfendale (1980).

Spectral correlations that have very uncertain significance but again are interesting have been suggested by Stanev et al., (1995) between the arrival directions of the highest energy events and 3C134 (with unknown redshift), Cygnus-A, NGC 315, 3C31, and M87.

\section{Gamma Ray Bursts and UHECRs}

A few papers have been written recently on the possible association of the source of UHECRs with gamma ray burst sources of cosmological origin (Waxman 1995, Vietri 1995, Milgram and Usov 1995a,b). After brushing aside the initial reaction that this is merely equating the two most un- 
known sources in high-energy astrophysics, one realizes that the proposal is based on two interesting coincidences of the flux and energy in UHECRs calculated from the standard Mezaros and Rees (1994) model. Relativistic Fireball models for cosmological gamma ray bursts are based on a millisecond rise time, corresponding to a scale of $\sim 10^{7} \mathrm{~cm}$ and a cosmological burst luminosity of $\sim 10^{51} \mathrm{erg} \mathrm{s}^{-1}$ over the burst lifetime of order of seconds. The resulting hyper-relativistic shocks can be used to accelerate particles to energies of order $\sim 10^{20} n \Theta^{-5 / 3} \mathrm{eV}$ where $n$ is the density of the ambient ISM and $\Theta$ is the beaming semi-opening angle (Vietri 1995). This is standard ping-pong shock acceleration achievable in just a few hits with a spectrum tending toward $\mathrm{E}^{-2}$. For a high efficiency of production of UHECRs such that, not uncommonly, the energy density in UHECRs is a significant fraction of the ram pressure of the shock, the calculated flux in UHECRs is quite consistent with the observed flux of $\sim 3 \times 10^{-8} \mathrm{Mpc}^{-3} \mathrm{yr}^{-1}$.

UHECRs are scattered through $\sim 5^{0} Z \quad E_{20}^{-1} \quad R_{10 \mathrm{Mpc}} B_{-9}$ by a coherent magnetic field and $\sim 1^{0} Z \quad E_{20}^{-1} \quad R_{10 \mathrm{Mpc}}^{1 / 2} l_{\text {coherence, } 1 \mathrm{Mpc}}^{1 / 2} B_{-9}$ for an incoherent structure that they diffuse through. Thus the time delay for a pulse from a GRB at $\sim 100 \mathrm{Mpc}$ is greater than $\gtrsim 10^{6} \mathrm{yr}$. Therefore no close temporal association should be observed.

\section{Events Above 100 EeV: Is There New Physics?}

The events above $100 \mathrm{EeV}$ are rare and can arise from special local sources. They may even be made of Fe since there is nothing known about their composition. If one takes the point of view that they are a hard component above the GZK cut-off then a question of new physics arises.

A conventional second phase statistical acceleration model calculated by NMA was shown to fail because the acceleration at known sources was insufficient to overcome the losses incurred traveling between acceleration sites. I strongly believe that this type of model could work and only currently fails due to our lack of knowledge of the structure and energy sources in the KM. In fact, the IGM probably has a structure as rich as the ISM. I expect the IGM is pervaded by a rich system of winds, explosions, shocks, structure formation etc. resulting from QSOs, galaxy formation, star burst galaxies, AGNs etc. This structure could produce UHECR spectrum without difficulty. This is probably the most important, albeit speculative, point of this lecture.

An alternative and interesting point of view is that the events above the GZK cut-off herald new physics (Sigl et al., 1995). There is a gap in the data of half a decade in energy between the two highest events and the rest of the data. Future experiments will establish if it is significant. If the gap persists then new physics may be involved. Top-down scenarios where the UHECRs 
are products of the flat injection spectrum of decaying objects associated with the GUT epoch. Foregoing sentence needs to be corrected for syntax Topological defects have been proposed as a possibility here (Bhattacharjee et al., 1992). Waxman (1995) produces a spectrum in which it does indeed look like the two highest energy events are special.

It is a pleasure to thank my collaborators in the UHECR work, A. Achterberg and D. Melrose for their advice, encouragement, and very stimulating scientific discussions throughout the course of this ongoing work. There were also very useful and interesting discussions on these and related subjects with T. Gaisser, C. Lacey, R. Protheroe, M. Rees, J. Stanev, M. Vietri and A. Waxman.

\section{References}

Axford, W. I. 1991, Ap. J. Suppl., 80, 937

Bhattacharjee, P., Hill, C. T., and Schramm, D. N. 1992, Phys. Rev. Lett, 69, 567

Bird, D. J. et al., 1993, Phys. Rev. Lett., 21, 3401

Cavallo, G 1978, $A \& A, 65,415$

Cesarsky, C. and Ptuskin, V. 1992, Nucl. Phys. B.(Proc. Suppl.), 28B

Donahue, M., Aldering, G. and Stocke, J.T. 1995, Ap. J., 450, L45

Done, C. and Fabian, A. 1989, 240, 81

Gaisser, T., Halzer, F., and Stanev, T. 1995, Phys. Reports, 258, 174

Gaisser, T. et al., 1993, Phys. Rev. P, 47, 1919

Giler, M., Wdowczyk, J., and Wolfendale, A. W. 1980, J. Phys. G. Nucl. Phys., 6, 1561

Hill, C. T. and Schramm, D. N. 1985, Phys. Rev. D, 31, 564

Hillas, A. M. 1984, Ann. Rev. A. A., 22, 425

Jokipii, J. R. and Morfill, G. E. 1985, Ap. J. Lett, 290, L1

Kang, H., Ryu, D., and Jones, T. W. 1996, Ap. J. Lett, in press

Mannhein, K. Astroparticle Physics, 3, 295

Mezaros, P. and Rees, M. J. 1994, M.N.R.A.S., 269, L41

Milgrom, M. and Usov, V. 1995a, preprint

Milgrom, M. and Usov, V. 1995b, preprint

Norman, C. and Lacey, C. 1995, Ap. J., in preparation

Norman, C. A., Melrose, D. B., and Achterberg, A. 1995, Ap. J., November 20, in press (NMA)

Protheroe, R. J. and Szabo, A. P. 1992, Phys. Rev. Lett., 69, 2885

Rachen, G. P., Stanev, T., and Biermann, P. L. 1993, Astro. Ap., 273, 377

Rees, M. J. 1987, M.N.R.A.S., 228, 47

Sigl, G., Lee, S., Schramm, D. N., and Bhattacharjee, P. 1995, Science, in press

Sigl, G., Schramm, D. N., and Bhattacharjee, P. 1995, Astroparticle Physics, in press

Stanev, T., Biermann, P. L., Lloyd-Evans, J., Rachen, J. P., and Watson, A. 1995, Phys. Rev. Lett, in press

Stecker, F. W., Done, C., Salamon, M. H., and Sommers, P. 1991, Phys. Rev. Lett., 66, 2697

Szabo, A. P. and Protheroe, R.J. 1994, Astroparticle Physics, 2, 375

Vietri, M. 1995, Ap. J., in press

Waxman, E. 1995, Ap. J. Lett., in press

Waxman, E. 1995, Phys. Rev. Lett, 75, 386 